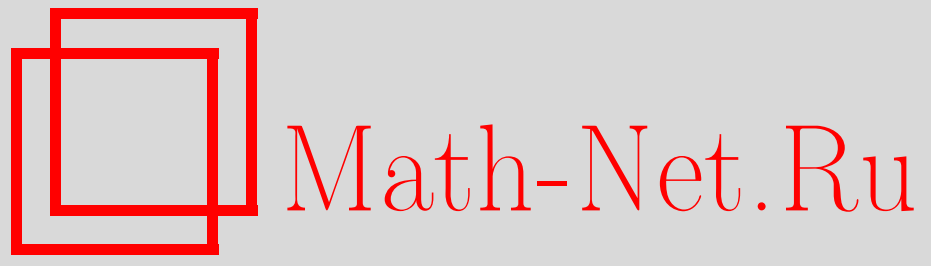

Общероссийский математический портал

А. В. Колесников, О непрерывных образах меры Лебега, Матем. заметки, 1999, том 65, выпуск 5, 790-793

DOI: https://doi.org/10.4213/mzm1112 
Использование Общероссийского математического портала Math-Net.Ru подразумевает, что вы прочитали и согласны с пользовательским соглашением http://www. mathnet.ru/rus/agreement

Параметры загрузки:

IP: 3.81 .55 .215

26 апреля 2023 г., 03:12:58

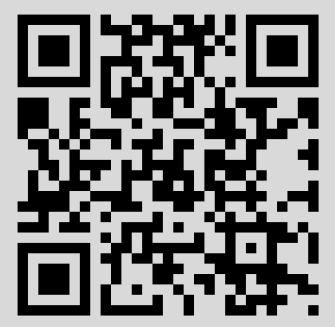




\section{О НЕПРЕРЫВНЫХ ОБРАЗАХ МЕРЫ ЛЕБЕГА}

\section{А. В. Колесников}

В зтой заметке изложено решение задачи описания мер, являющихся непрерьвным образом меры Лебега на $[0,1]$. Эта задача была поставлена П. В. Парамоновым в связи с некоторьми вопросами теории оптимального поиска (см. [1], [2]). Результат состоит в том, что вероятностная борелевская мера в топологическом пространстве является непрерывным образом меры Лебега на $[0,1]$ в точности тогда, когда ее носитель есть связный и локально связный компакт (что, как известно, равносильно тому, что этот носитель есть непрерывный образ отрезка).

Напомним сначала некоторые определения из теории меры и общей топологии. Пусть $(\Omega, \mathcal{A}, \mu)$ - пространство с мерой, $(E, \mathcal{B})$ - измеримое пространство, $\xi: \Omega \mapsto E$ - измеримое отображение. Равенство

$$
\mu \circ \xi^{-1}(B)=\mu\left(\xi^{-1}(B)\right), \quad B \in \mathcal{B}
$$

определяет меру на $\mathcal{B}$. Мера $\mu \circ \xi^{-1}$ называется образом меры $\mu$ при отображении $\xi$.

Пусть $\mu$ - борелевская вероятностная мера на топологическом пространстве. Пусть $S_{\mu}-$ пересечение всех замкнутых подмножеств полной меры. Если $\mu\left(S_{\mu}\right)=1$, то $S_{\mu}$ назьвается носителем меры $\mu$. Известно, что в полном сепарабельном метрическом пространстве каждая борелевская вероятностная мера обладает носителем; более того, всякая мера Радона обладает носителем (см. [3, предложение 3.4 , с. 36]).

Работа написана при поддержке Российского фонда фундаментальных исследований, гранты № 96-15-96865, № 97-01-00932, и российско-германского гранта. 
Топологическое пространство называется локально связным в точке $x$, если всякая открытая окрестность точки $x$ содержит некоторую ее связную окрестность (здесь и далее под окрестностью точки $x$ подразумевается любое множество, содержащее некоторое открытое подмножество $V$, причем $x \in V$ ). Топологическое пространство назьвается локально связным, если оно локальн связно в каждой своей точке.

ЗАмЕчАниЕ 1. Известно, что образ отрезка при непрерывном отображении является метризуемым компактом (см. [4, теорема 4.4.15]). Кроме того, метрический компакт является непрерывным образом отрезка в точности тогда, когда он связен и локально связен (см. $[4,6.3 .14])$.

ТЕОРема 1. Пусть $K$-метрический компакт, являющийся образом отрезка $[0,1]$ при непрерывном отображении $f, \mu$-борелевская вероятностная мера на $K$, причем $K-e е$ носитель. Тогда существует такое непрерывное сюрвективное отобрахение $g:[0,1] \mapsto$ $K$, что $\mu=\lambda \circ g^{-1}$, где $\lambda$ - мера Лебега на отрезке $[0,1]$.

ДоКАЗАТЕЛЬСтво следует из леммм 2,3 и 4 , изложенных ниже.

Всюду ниже сохраняются обозначения, введенные в теореме.

ЛЕмма 1. У любой точки $y \in K$ существует сколь угодно малая окрестность $U$, являющаяся непрерывным образом отрезка.

ДокАЗАТЕЛЬСтво. Пусть $A_{k}=[k / n,(k+1) / n], k=0,1, \ldots n-1$. Положим $U=$ $\bigcup_{k: y \in f\left(A_{k}\right)} f\left(A_{k}\right)$. Очевидно, $U$ - непрерывный образ отрезка. Это множество - окрестность, так как вместе с $y$ оно содержит открытое множество $K \backslash \bigcup_{k: y \notin f\left(A_{k}\right)} f\left(A_{k}\right)$. В силу равномерной непрерьвности $f$ и неравенства треугольника окрестность $U$ можно сделать сколь угодно малой, увеличивая $n$.

Лемма 2. Существует такое непрерывное сюрвективное отобрахение

$$
\varphi:[0,1] \mapsto K
$$

что $\mu(\varphi(V)) \neq 0$ для любого открытого $V$ из $[0,1]$.

ДоКАЗАТЕЛЬСТво. Будем строить $\varphi$, исходя из отображения $f$. Пусть $V_{0}$ - объединение всех таких открытых множеств, которые $f$ переводит в множество меры нуль. Имеем $V_{0}=\bigcup i=1^{\infty} J_{i}$, где $J_{i}=\left(a_{i}, b_{i}\right)$, либо $J_{i}=\left[0, b_{i}\right)$, либо $J_{i}=\left(a_{i}, 1\right]$, причем $J_{i} \cap J_{j}=\varnothing$, если $i \neq j$. Считаем, что длина $J_{i}$ не возрастает с ростом $i$. Пусть $m$ - наименьшее натуральное число такое, что существуют такие $J_{1}, J_{2} \ldots J_{k_{1}}$, что длина каждого из них не меньше $1 / 2^{m}$. Пусть $c_{1}-$ середина интервала $J_{1}$. По предыдущей лемме найдем окрестность $U_{1}$ точки $f\left(c_{1}\right)$, являющуюся непрерывным образом отрезка, с диаметром меньше чем $1 / 2$ (в метрике $K$ ). Кроме того, найдется $\delta_{1}$ такое, что $\left(c_{1}-\right.$ $\left.\delta_{1}, c_{1}+\delta_{1}\right)$ лежит в $J_{1}$ и $f\left(c_{1}-\delta_{1}, c_{1}+\delta_{1}\right) \subset U_{1}$. Построим такое непрерьвное отображение $f_{1}$ отрезка $\left[c_{1}-\delta_{1}, c_{1}+\delta_{1}\right]$ на $U_{1}$, что

$$
f_{1}\left(c_{1}-\delta_{1}\right)=f\left(c_{1}-\delta_{1}\right), \quad f_{1}\left(c_{1}+\delta_{1}\right)=f\left(c_{1}+\delta_{1}\right) .
$$

Аналогично для всех $1 \leq i \leq k_{1}$ строим отображения $f_{i}$ в $\delta_{i}$-окрестностях точек $c_{i}$, где $c_{i}$ - середина $J_{i}$. Пусть отображение $\varphi_{1}$ совпадает с $f$ вне этих окрестностей, а на них совпадает с $f_{i}$; тогда $\varphi_{1}$ непрерывно, причем $\rho\left(f, \varphi_{1}\right) \leq 1 / 2$, где $\rho$-равномерная метрика. Наибольшее открытое множество, которое $\varphi_{1}$ переводит в множество меры нуль, не содержит интервалов длины большей чем $1 / 2^{m}$, так как $\mu\left(U_{i}\right) \neq 0$, где $1 \leq i \leq k_{1}$. Выделим теперь интервалы $J_{k_{1}+1}, J_{k_{1}+2} \ldots J_{k_{2}}$, длина каждого из которых болше чем $1 / 2^{m_{1}}$ для некоторого $m_{1}>m$. Аналогично строим непрерьвное отображение $\varphi_{2}$ с условием $\rho\left(\varphi_{1}, \varphi_{2}\right) \leq 1 / 4$. Проведя эти построения конечное или счетное число раз, получим последовательность непрерывных отображений $\varphi_{n}$, причем $\rho\left(\varphi_{n}, \varphi_{n+1}\right) \leq 1 / 2^{n+1}$. В пределе получаем непрерьвное отображение $\varphi$. Пусть $X_{0}=[0,1] \backslash V_{0}, X_{i}=[0,1] \backslash V_{i}$, где $V_{i}$ - наибольшее открытое множество, которое $\varphi_{i}$ переводит в множество меры нуль. Так как по построению каждое из $\varphi_{j}$, где $j>i$, совпадает на $X_{i}$ с $\varphi_{i}, \mathrm{a} \bigcup i=1^{\infty} X_{i}$ всюду плотно на $[0,1]$, то $\varphi$ переводит открытые множества в множества положительной меры. Далее, $\varphi[0,1]=K$, посколку уже $f\left(X_{0}\right)=K$ (иначе сушествовало бы открытое множество в $K \mu$-меры нуль). 
Лемма 3. Пусть $X$ и $K$-метрические компакты, $f: X \mapsto \mathrm{K}$ сюрвективное непрерывное отобрахение метрических компактов и $\mu$-борелевская вероятностная мера на $K$. Тогда существует такая борелевская вероятностная мера $\nu$ на $X$, ито $\nu \circ f^{-1}=\mu$. Ecли $\mu(f(W)) \neq 0$ для любого открытого $W$ в $X$, то можсно выбрать $\nu$ такой, что ее носитель совпадает со всем $X$.

ДокАЗАТЕЛЬСтво. Первое утверждение хорошо известно (см., например [5, теорема 6.14]). Оно доказывается следуюшим образом. В пространстве $C(X)$ рассмотрим подпространство, состоящее из функций вида $\psi \circ f, \psi \in C(K)$. На нем определим линейный функционал $L$ по формуле

$$
L(\psi \circ f)=\int_{K} \psi(y) \mu(d y) .
$$

Норма этого функционала равна единице. По теореме Хана-Банаха продолжим его на все $C(X)$ с сохранением нормы. По теореме Рисса существует такая знакопеременная борелевская мера $\nu$ на $X$, что для всех непрерьвных $\varphi \in C(X)$

$$
L(\varphi)=\int_{X} \varphi(x) \nu(d x)
$$

В частности, для всех непрерьвных $\psi \in C(K)$

$$
\int_{X} \psi(f(x)) \nu(d x)=\int_{K} \psi(y) \mu(d y) .
$$

Для $\psi=1$ получаем, что $\nu(X)=1$. Теперь если $\nu=\nu_{+}-\nu_{-}-$разложение $\nu$ на положительную и отрицательную части, то в силу того, что норма $L$ равна единице, имеем $\nu_{+}(X)+\nu_{-}(X)=$ 1. Отсюда видно, что мера $\nu$ неотрицательна. Соотношение (1) означает, что меры $\nu \circ f^{-1}$ и $\mu$ приписывают равные интегралы ограниченным непрерывным функциям, что влечет равенство $\nu$ 。 $f^{-1}=\mu$.

Докажем второе утверждение. Рассмотрим счетную базу окрестностей $\left\{W_{i}\right\}$ в $X$. Так как $\mu\left(f\left(W_{i}\right)\right) \neq 0$, существует такой компакт $K_{i} \subset W_{i}$, что $\mu\left(f\left(K_{i}\right)\right) \neq 0$ (так как любое открытое подмножество метрического компакта является счетньп объединением компактов). На $K_{i}$ построим меру $\nu_{i_{1}}$, которая является прообразом меры $\left.\mu\right|_{f\left(K_{i}\right)}$, т.е. $\nu_{i_{1}}$ - такая неотрицательная мера на $K_{i}$, что $\nu_{i_{1}} \circ f^{-1}=\left.\mu\right|_{f\left(K_{i}\right)}$. На $X$ выберем меру $\nu_{i_{2}}$, которая является прообразом меры, равной нулю на $f\left(K_{i}\right)$ и совпадающей с мерой $\mu$ на $K \backslash f\left(K_{i}\right)$. Положим $\nu_{i}=\nu_{i_{1}}+\nu_{i_{2}}$. Имеем $\nu_{i} \circ f^{-1}=\mu$, причем $\nu_{i}\left(W_{i}\right) \neq 0$. Положим

$$
\nu=\sum_{i=1}^{\infty} \frac{1}{2^{i}} \nu_{i}
$$

Очевидно, что носитель $\nu$ совпадает с $X$ и

$$
\nu \circ f^{-1}=\sum_{i=1}^{\infty} \frac{1}{2^{i}} \nu_{i} \circ f^{-1}=\sum_{i=1}^{\infty} \frac{1}{2^{i}} \mu=\mu .
$$

ЛЕмма 4. Любая борелевская вероятностная мера $\nu$ на отрезке [0,1] с носителем $[0,1]$ является образом меры Лебега на $[0,1]$ при некотором непрерывном сюрдективном отобрахсении $s:[0,1] \mapsto[0,1]$.

ДоКАЗАТЕЛЬСТво. Если $F(t)=\nu([0, t))$ - функция распределения, то искомое отображение задается формулой

$$
s(t)=\sup _{x \in[0,1]}\{x: F(x) \leq t\} .
$$


СлЕдствиЕ. Непрерывные образы меры Лебега на $[0,1]$ - это вероятностные борелевские меры, носители которых являются связными, локально связными компактами.

ЗАмЕч АниЕ 2. В качестве простого следствия получаем, что непрерывные образы меры Лебега на $[0,1]^{n}$ - это вероятностные борелевские меры, носители которых являются связными, локально связньми компактами.

В зак лючение отметим, что П. В. Парамоновым были поставлены вопросы об описании образов меры Лебега при более специальных отображениях (например, непрерывно дифференцируемых заданного порядка). Эти вопросы остаются пока открытыми.

Автор благодарен П. В. Парамонову за постановку задачи и плодотворное обсуждение, а также В.И. Богачеву за полезные замечания.

\section{СПИСОК ЦИТИРОВАННОЙ ЛИТЕРАТУРЫ}

1. Парамонов П.В.// Автоматика и телемеханика. 1988. №11. С. 102-112. 2. Парамонов П.В. Исследование на экстремум интегрального функционала, связанного с задачей оптимального поиска // Тезисы докладов Международной конференции по комплексному анализу и смежным вопросам. Нижний Новгород, Июнь, 1997. С. 55-56. 3. Вахания Н. Н., Тариеладзе В. И., Чобанян С. А. Вероятностные распределения в банаховых пространствах. М.: Наука, 1985. 4. Энгелькинг Р. Общая топология. М.: Мир, 1986. 5. Богачев В. И. Меры на топологических пространствах / / Итоги науки и техники. Современная математика и ее приложения. Т. 36. М.: ВИНИТИ, 1996.

Московский государственный университет им. М.В. Ломоносова

Поступило 08.04 .98 